\title{
The Comparison of learning radiographic extraoral landmarks through two methods
}

Tahmineh Razi ${ }^{1}$, Alireza Sighari Deljavan ${ }^{2 *}$

1. Assistant Professor, Department of Oral and Maxillofacial Radiology, Faculty of Dentistry, Tabriz University of Medical Sciences, Tabriz, Iran

2. Post graduate Student, Department of Oral \& Maxillofacial Surgery, Isfahan (Khorasgan) Branch, Islamic Azad University, Isfahan, Iran

\begin{abstract}
Objectives: The long time interval between teaching anatomy courses to students and the courses on extraoral radiographs results in forgetting the location of radiographic anatomic landmarks without any retention; therefore, it is necessary to use other educational techniques due to short duration of educational courses. To this end, the present study was undertaken to evaluate the effect of a combination of lectures and computer techniques on teaching extraoral radiographs to undergraduate dental students. Methods: In the present interventional study, dental students entering the university in the same year (referred to as one class) were divided into two groups; one group received conventional teaching and one group received combinational teaching technique. Students entering the next year were taught using the same teaching techniques. The results were compared between groups and classes. Results: Comparison of the results of the first test between the two classes showed significant differences between the two groups $(P<0.001)$. Comparison of the results of the second test between the two classes revealed no significant differences in the conventional method $(P=0.76)$; however, the combination technique exhibited significant differences $(P=0.006)$. In the first test, comparison of the two techniques in the students entering the university in 2006 showed no significant differences $(P=0.055)$; however, students entering the university in 2007 exhibited significant differences $(P=0.03)$. In the second test, comparison of the two techniques in both classes revealed significant differences $(P<0.001)$.

Conclusion: If more time is allocated to the combination technique, learning improves even in a short time. This method can replace the conventional method to teach interpretation of extraoral radiographs after evaluation of academic level of the studies.
\end{abstract}

Keywords: Computer-assisted learning; extra-oral radiography; lecture; 


\section{Introduction}

Extraoral radiographs are usually ordered by general dental practitioners. These radiographs are wanted in some special cases such as evaluation of sinuses, fractures and evaluation of lesions (1). In order to diagnose abnormal conditions on radiographic images, the practitioner should have a sound knowledge about the normal radiographic anatomy. Teaching interpretation of extraoral radiographs to dental students is carried out very briefly during a very limited period of time with the use of slides, which includes introducing radiographs and indications for ordering them. A proper understanding of various anatomic structures and regions requires a thorough understanding about the anatomy and the radiographic views of these areas from different points (lateral, anteroposteior etc). Three-dimensional understanding of the skull and maxillofacial region is very useful for the diagnosis of fractures, lesions etc. Given what was discussed above, the time allocated to teach interpretation of extraoral radiographs to dental students is very short and the time interval between teaching anatomy (as a prerequisite for interpreting extraoral radiographs) and the extraoral radiographic image interpretation courses is very long. Therefore, the anatomy lessons are forgotten and the radiographic landmarks are not learned very well and are not retained. Therefore, due to limited educational hours allocated to these subjects, it is necessary to use other techniques to overcome problems related to learning these lessons; in this context, use of computer techniques might promote learning (2). The World Wide Web has been introduced as a rich source for education and learning processes in the fields of medicine and nursing (2). A large number of studies have been carried out on computer-assisted learning (CAL). In the majority of these studies, no statistically significant differences have been found between study groups in relation to comparison of different commonly used lecturing techniques (3-5).

In different branches of dentistry, including periodontology, orthodontics, morphology and endodontics, similar results have been achieved (6-9). No significant relation has been established between the time spent on CAL and students' scores $(4,9)$. However, advantages, such as motivation for learning, freedom of action and a feeling of security by the students, have been reported $(3,10)$, which have resulted in their satisfaction with CAL method despite lack of differences in the results of comparisons with lecture-based teaching techniques $(9,11)$. In some studies, female students have preferred lectures $(6,9,12)$ and male students have preferred self-learning methods (9). Studies have not suggested that CAL method should replace lecture-based method (2,9); rather, they have suggested a combination of lectures and $\operatorname{CAL}(3,6,7,13)$. In dental radiology courses on interpretation of extraoral radiographs, CAL method has been reported to be effective but no relationship has been found with retention (11). No significant findings have been reported in relation to learning of clinical courses $(10,11)$. In learning courses such as surgery and radiology, there is a need to learn basic sciences and gain a 3dimentional understanding of body structures so that real learning would be achieved in order to interpret and react properly and correctly. In this context, use of 3-dimensional computer images is useful (14); however, it is necessary to combine teaching of basic sciences and other courses (15). It appears that separating basic science courses is not important; however, these courses help students learn diseases and their characteristics better (15). Considering the time interval between teaching anatomy courses, as prerequisites for understanding 
radiographic images, it is necessary to teach interpretation of extraoral radiographs with refresher courses of anatomy. In addition, a method should be introduced so that after teaching the lessons, in information will be retained for clinical applications after graduation from the university.

The aim of the present study was to provide a better understanding and greater retention of anatomic landmarks on extraoral radiographs. Therefore, considering the results of previous studies on the effect of adjunctive techniques and suggestions regarding not replacing conventional methods with adjunctive methods and satisfaction of students with the advantages of CAL, a combination of lecturebased method and CAL were evaluated in relation to teaching the interpretation of extraoral radiographs to undergraduate dental students.

\section{Methods}

The present interventional study was carried out in 2009. The subjects consisted of 50 dental students in the 6th semester of their studies. The students had taken theoretical radiology course II on interpretation of extraoral radiographs. The students were randomly divided into two groups. The dividing procedure was carried out by a university lecturer not involved in the teaching process. All the students signed an informed written consent form to take part in the study and attended the relevant classes based on the classification carried out. Group 1 consisted of 25 students who were taught by lectures in the classroom using the Microsoft Power Point program. The slides used consisted of images and texts used in the relevant reference books and the anatomic landmarks were shown and taught on slides prepared from extraoral radiographs. At the end of the course the students took a test, during which they were given a table consisting of 40 squares and were asked to record the name of the landmarks indicated on the radiograph in each square. The images had been selected from the textbook of radiography 1 , which had been used during the teaching. The images were appropriate for the research purpose and 5 university staffs in the field of oral radiology evaluated the validity of the images and their opinions were used to make the necessary modifications. In the present study each correct answer received a score of 1 and no scores were given to wrong answers. Classes for group 1 were held before the combination classes.

Group 2 consisted of 25 students, who were given a $C D$ on the anatomy lessons one week before the classes were held in order to refresh anatomy lessons. The students were asked to review the $C D$ before the classes began. The $C D$ contained a Microsoft Power Point program and each extraoral radiograph had been included in one program; each program consisted of radiographic images of one type of extraoral radiograph. The $C D$ also consisted schematic representations of dry skulls and corpses from human anatomy atlas books conforming to the type of the extraoral image involved. The procedures above were followed in order to refresh anatomy lessons of previous years and to compare them with different anatomic landmarks on radiographic images. In addition to reviewing the $C D$ provided, the students were asked to study the relevant landmarks on a dry skull. On The first class day the students were given a test to evaluate their knowledge about anatomy at baseline. The lecturer and postgraduate students in the Department of Maxillofacial Radiology asked the undergraduate students some questions about anatomic landmarks on a dry skull and the number of correct answers was recorded. This test was not one of the aims of the present study and was only carried out to classify students, appropriate to their basic knowledge, in teaching groups. Based on the results of this test, the students were divided into 3 groups of 
weak, moderate and strong. The students were divided into 4-student groups and in each group, weak, moderate and strong students were placed so that a strong student would be involved in the teaching process in each group. The groupings were carried out randomly so that a uniform distribution of students would be carried out in all the groups as far as possible. It was expected that in this way all the groups could progress with the same quality during teaching. The combination method was carried out in the Anatomy Hall of the Faculty of Medicine. Each 4-member group was given a laptop computer and a number of dry skulls. In order to help use the skull and change the slide in the laptop computer parallel with the lecture given by the professor, one postgraduate student of oral radiology was allocated to each group. The professor began to give the lecture and during the teaching, guidelines were provided for the groups by postgraduate students on how to use the slides and dry skulls. In this method, presentation of anatomic landmarks was not confined only to the slides similar to the lectures in group 1; rather the postgraduate students helped the undergraduate students compare the landmarks on the dry skull with those on radiographs. After the teaching period, a test was given to the students similar to that in group 1. Lectures were presented by two assistant professors in the Department of Maxillofacial Radiology. The time and content of the lectures and the slides presented were similar in both groups and based on the educational curriculum.

In order to evaluate retention of knowledge the second test was given 6 months after the first test to the students of both groups, without a prior warning, similar to the first test and with similar times in both groups. None of the groups had received further educations after the first test. In order to satisfy the students in relation to the study, after the second test, the first group underwent the combination educational method.

Students' demographic data and grades were kept confidential. The study design which was in accordance with the Helsinki Declaration of Human Rights was submitted to and approved by the Committee for Ethics in Research on Humans at Tabriz University of Medical Sciences (Ref number: 7524). After the study, the students were asked to give their opinion about the two teaching methods. The students in the second group were fully satisfied with the combination method and requested that the duration of teaching with the combination method be increased. Therefore, the same teaching technique was used for the 50 students entering the university the following year. However, the teaching duration increased up to 1 hour.

\section{Statistical analysis}

The data was analyzed using the SPSS software (version 16). Descriptive statistics including means and standard deviations were calculated for all variables and two-factor ANOVA were also used. In addition, Bonferroni post hoc test was used to make comparisons between the two groups. $\mathrm{P}<0.05$ was considered statistically significant.

\section{Results}

The means of the grades in both conventional and combinational groups for students of both classes (entering the university in 2006 and 2007) are presented in Table 1.

Comparison of the results of the first test between the two classes, in both conventional and combination groups, revealed statistically significant differences $(P<0.001)$. No statistically significant differences were observed between the two classes in the conventional method when the results of the second test were 


\begin{tabular}{lll}
\hline & Entering in 2006 & Entering in 2007 \\
\hline $\mathrm{T}_{1}$; the first test of the conventional group & $21.36 \pm 7.09$ & $13.23 \pm 4.49$ \\
$\mathrm{~T}_{2}$; the second test of the conventional group & $13.48 \pm 6.39$ & $14.04 \pm 6.64$ \\
$\mathrm{~T}_{1} ;$ the first test of the combinational group & $25.88 \pm 9.25$ & $16.38 \pm 6.41$ \\
$\mathrm{~T}_{2}$; the second test of the combinational group & $20.92 \pm 8.61$ & $27.69 \pm 8.28$ \\
\hline
\end{tabular}

Table 1: The means of grades in both tests in the two classes

compared $(P=0.76)$; however, there were significant differences in the results of the second test between the two classes in the combination method $(P=0.006)$.

The first test did not exhibit any significant differences between the conventional and combination methods in students entering the university in 2006 ( $P=0.055)$; however, there were significant differences in students entering the university in $2007 \quad(P=0.03)$. In relation to the second test, there were significant differences between the conventional and combination methods in both classes (2006 and 2007) $(P<0.001)$.

Figures 1, 2 and 3 show the error bars of the tests in both classes. In addition, the results of two-factor ANOVA showed that the cumulative effect of the two variables of the teaching technique and the entrance year on the results of retention tests was statistically significant $(P=0.04, F(1,98)=4.31)$.

\section{Discussion}

In the present study two conventional and combinational teaching methods were compared between the students of one class and also between the students of two classes whose students had entered the university with an interval of one year. All the conditions in the two classes and in the groups receiving different teaching techniques were the same. The only difference was the fact that upon the request of the students in the first class the combinational teaching technique in the second class was increased to two hours.

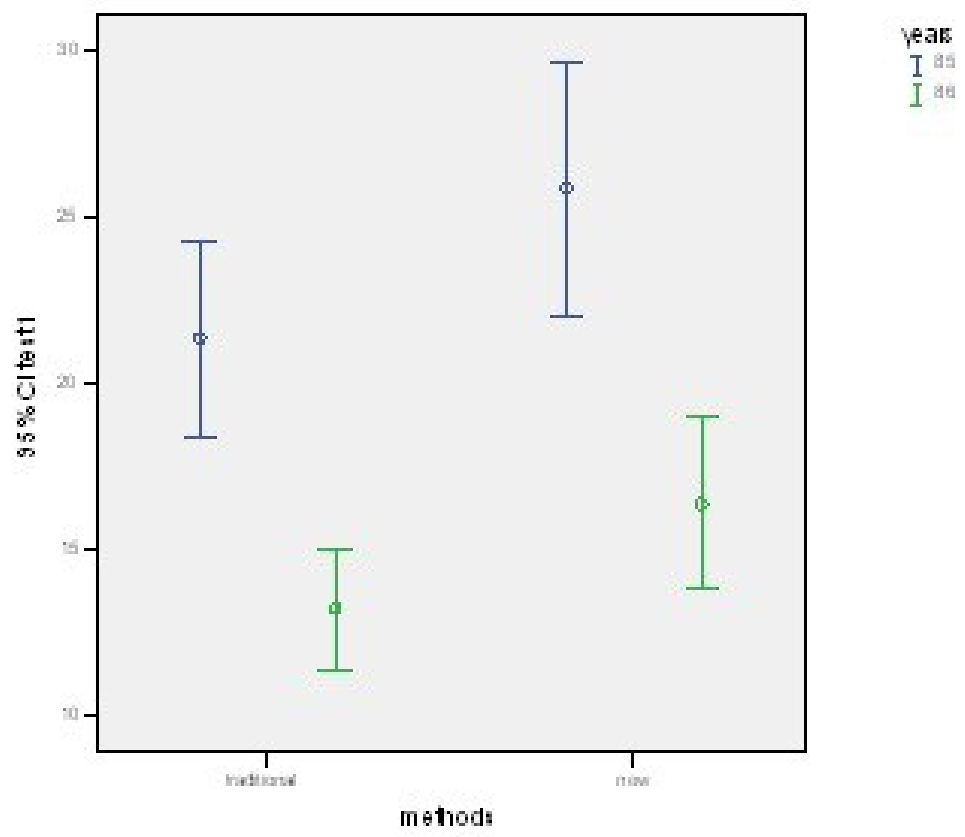

Figure 1: Comparison of Methods based on Year 


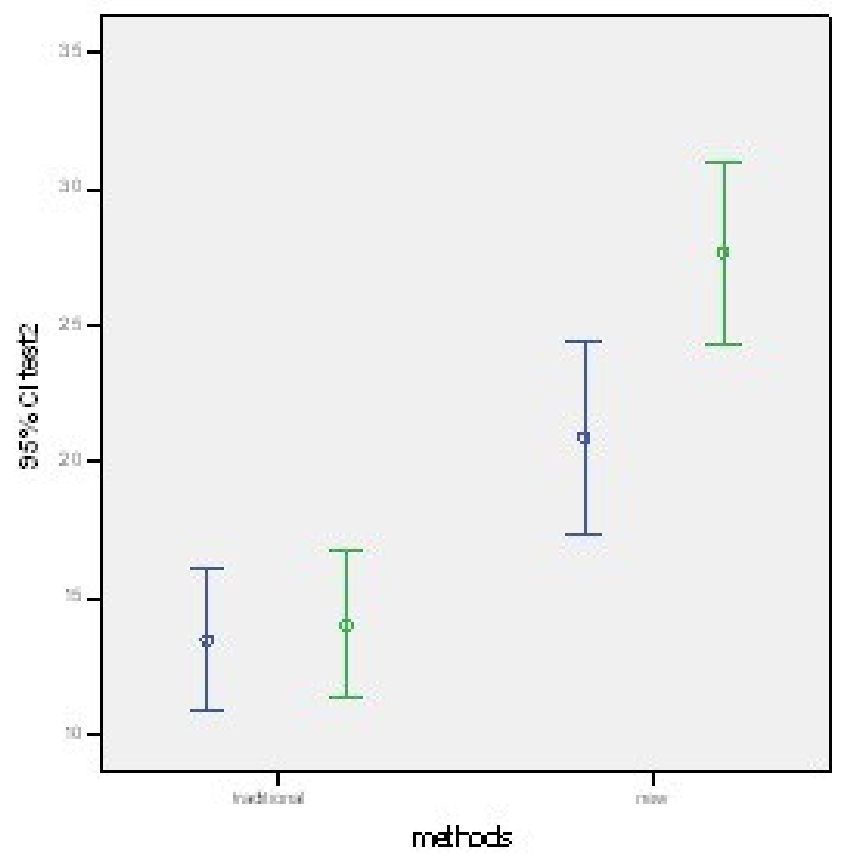

I

$\bar{I}$ a

Figure 2: Comparison of Methods based on Year

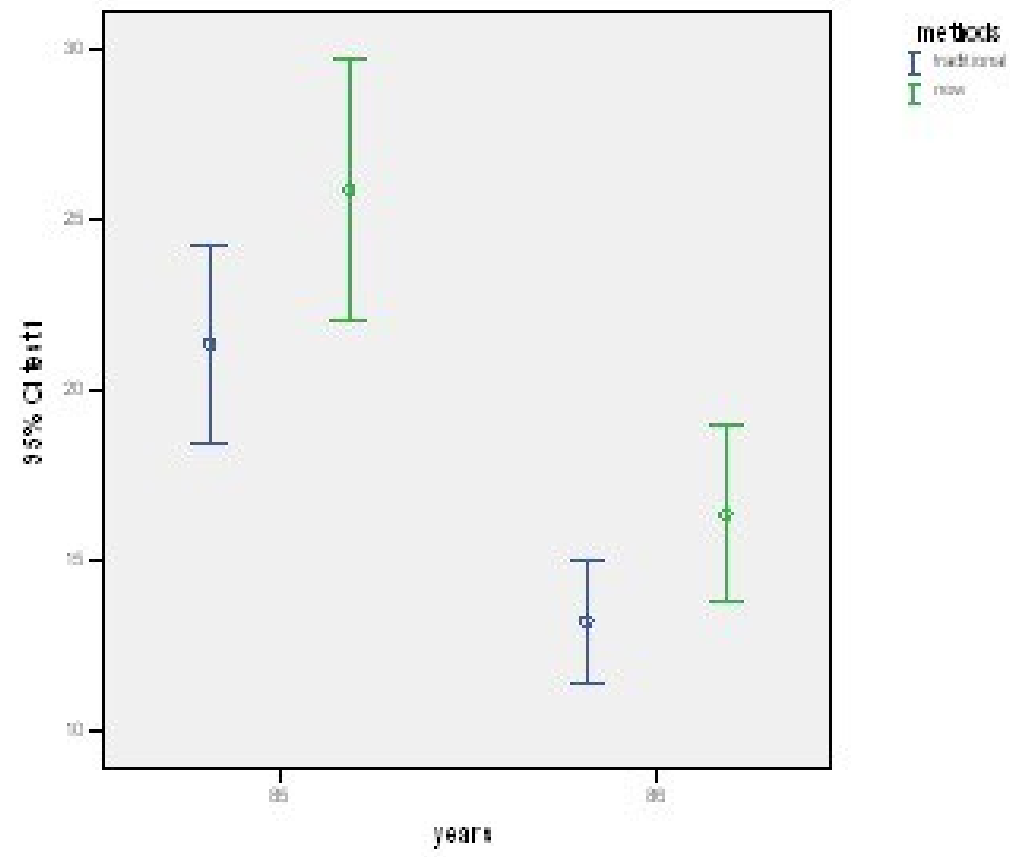

Figure 3: Comparison of years based on Methods 
The time spent on CAL is widely different based on the students' knowledge at baseline ad their interest in using such a technique. In orthodontic field the mean was reported to be 41-360 minutes (16). In the present study, a CD was given to the students one week before the combinational classes were held, which was similar in both classes (2006 and 2007).

Comparison of the results of the first test between the students entering the university in 2006 and 2007 in both conventional and combinational groups showed statistically significant differences. The mean grades of students entering the university in 2006 was higher than those of students entering the university in 2007, demonstrating a higher basic knowledge in the first class compared to the second class. In relation to the second test, there were no significant differences between the groups with the use of the conventional technique; however, there were significant differences in the results of the second test with the use of the combinational technique between the two classes, demonstrating similarity of learning with the use of the conventional technique in two groups with different levels of basic knowledge. However, the combination technique yielded better results in students with a higher knowledge level. The mean of grades in the second test in the combination technique group of students entering in 2007 was higher than all the other means. The long duration of teaching might have been more efficacious in solving the students' problems by providing better chance for guiding the students by postgraduate students. This is similar to tutorials in which it is possible to increase the students' motivation by their participation in the learning process (17). The second test was given 6 months after the first test without students' prior knowledge and without their reviewing the lessons in order to evaluate retention of knowledge with CAL. Retention of knowledge is more valuable than short-term learning (18) because there is a long interval between presentation of radiography lessons and graduation from the university and application of knowledge in the clinic.

Comparison of the first test between the conventional and combinational techniques in students entering the university in 2006 exhibited no significant differences. The first test was given immediately after teaching with the two techniques in order to evaluate shortterm learning. A lack of significant differences between the two techniques reflects a lack of any effect of these techniques on short-term learning. However, in the case of students entering the university in 2007, the presence of significant differences in short-term learning, demonstrated by a higher mean grade in the combination group compared to the conventional group, reveals that if the duration of combinational technique increases, better learning is achieved even in the short-term. In addition, despite lack of significant differences in students entering in 2006, the students had a positive attitude toward CAL. Studies have shown that students have different attitudes toward CAL and evidence shows that they believe it is more time-saving (6). There are some other factors involved in satisfaction, including free review of the subjects and learning at any time the individual chooses.

The results in both classes (2006 and 2007) in relation to the comparison of combinational and conventional techniques in the second test within each class showed better results with the combinational technique, i.e. the combinational technique had resulted in better retention compared to the lecture technique in both classes. A higher mean of grades in the second test in students entering in 2007 with the combinational technique shows that it had resulted in better retention of knowledge in addition to better efficacy of CAL and a longer duration of teaching. 
However, there are some limitations with the use of the combinational technique, making it more time-consuming and costly for professors despite its efficacy in relation to the time and cost for students. Conversion of the face-to-face lecturing system to e-learning requires spending a long time on behalf of the universities (13) For example, a study showed that it is necessary to spend 300 hours to prepare a CAL program lasting one hour for the analysis of mixed dentition (16). In the present study, preparation of a $C D$, containing images scanned from different books and creation of effects in order to determine anatomic landmarks took two months. Nonetheless, there is limited evidence in relation to the efficacy of CAL for clinical abilities (6). Given the positive attitudes of students and despite the time-consuming and costly nature of preparing CAL programs, researchers believe that it is a necessary component for better learning (13).
However, the majority of studies have shown that it cannot replace lectures and texts.

The combinational technique, whether in the short term or long term, was superior to the conventional method in students with a higher basic knowledge level. The retention of knowledge in both classes was better with the combinational technique compared to the conventional method; therefore, it can replace the conventional method in teaching interpretation of extraoral radiographs. It is suggested that in future studies retention of knowledge be evaluated in graduates.

\section{Acknowledgements}

This study was supported and funded by Tabriz University of Medical Sciences. The authors would like to thank the staff at the Department of Oral Radiology for their assistance and students for participating in the study. The authors declare that they have no conflicts of interest.

\section{References}

1. White SC, Pharoah MJ. Oral Radiology and Interpretation. 6th ed. St. Louis: Mosby; 2009. pp 191

2. Walmsley D. CAL programs in orthodontics elicit positive responses from students. Evidence- Based Dentistry 2006;7:77.

3.Rosenberg H, Grad HA, Matear DW. The effectiveness of computer-aided, self-instructional programs in dental education: a systematic review of the literature. J Dent Educ 2003;67:524-32.

4. Jeffries PR. Computer versus lecture: a comparison of two methods of teaching oral medication administration in a nursing skills laboratory. J Nurs Educ 2001;40:323-9.

5. Jenkins S, Goel R, Morrell DS. Computer-assisted instruction versus traditional lecture for medical student teaching of dermatology morphology: a randomized control trial. J Am Acad Dermatol 2008;59:255-9.

6 .Al-Jewair TS, Qutub AF, Malkhassian G, Dempster LJ. A systematic review of computer-assisted learning in endodontics education. J Dent Educ 2010;74:601-11.

7. Nance ET, Lanning SK, Gunsolley JC. Dental anatomy carving computer-assisted instruction program: an assessment of student performance and perceptions. J Dent Educ 2009;73:972-9.

8. Bissell V, McKerlie RA, Kinane DF, McHugh S. Teaching periodontal pocket charting to dental students: a comparison of computer assisted learning and traditional tutorials. Br Dent J 2003;195:333-6.

9. Rosenberg H, Posluns J, Tenenbaum HC, Tompson B, Locker D. Evaluation of computer-aided learning in orthodontics. Am J Orthod Dentofacial Orthop 2010;138:410-9.

10. Ludlow JB, Platin E. A comparison of Web page and slide/tape for instruction in periapical and panoramic radiographic anatomy. J Dent Educ 2000;64:269-75. 
11. Howerton WB Jr, Platin E, Ludlow J, Tyndall DA. The influence of computer-assisted instruction on acquiring early skills in intraoral radiography. J Dent Educ 2002;66:1154-8.

12. Rosenberg H, Sander M, Posluns J. The effectiveness of computer-aided learning in teaching orthodontics: a review of the literature. Am J Orthod Dentofacial Orthop 2005;127:599-605.

13. Tan PL, Hay DB, Whaites E. Implementing e-learning in a radiological science course in dental education: a shortterm longitudinal study. J Dent Educ 2009;73:1202-12.

14. Davis J, Crabb S, Rogers E, Zamora J, Khan K. Computer-based teaching is as good as face to face lecture-based teaching of evidence based medicine: a randomized controlled trial. Med Teach 2008;30:302-7.

15. Baghdady MT, Pharoah MJ, Regehr G, Lam EW, Woods NN. The role of basic sciences in diagnostic oral radiology. J Dent Educ 2009;73:1187-93.

16. Al-Jewair TS, Azarpazhooh A, Suri S, Shah PS. Computer-assisted learning in orthodontic education: a systematic review and meta-analysis. J Dent Educ 2009;73:730-9.

17. Potomkova J, Mihal V, Cihalik C. Web-based instruction and its impact on the learning activity of medical students: a review. Biomed Pap Med Fac Univ Palacky Olomouc Czech Repub 2006;150:357-61.

18. Lieberman G, Abramson R, Volkan K, McArdle PJ. Tutor versus computer: a prospective comparison of interactive tutorial and computer assisted instruction in radiology education. Acad Radiol 2002;9:40-9. 